\title{
SUBLINEAR TRACKING IN THURSTON'S METRIC FOR RANDOM WALKS
}

\author{
Huiping PAN
}

\begin{abstract}
In this paper, we prove the sublinear tracking property in Thurston's metric for sample paths of random walks on mapping class group.
\end{abstract}

\section{Introduction}

Let $S$ be a closed surface of genus $g \geq 2$. A marked Riemann surface of genus $g$ is a pair $(X, f)$ consisting of a Riemann surface $X$ of genus $g$ and an orientation-preserving homeomorphism $f: S \rightarrow X$. Two marked Riemann surfaces $\left(X_{1}, f_{1}\right)$ and $\left(X_{2}, f_{2}\right)$ are equivalent if there exists a conformal map $h: X_{1} \rightarrow X_{2}$ in the homotopy class of $f_{2} \circ f_{1}^{-1}$. The Teichmüller space of $S$, denoted by $T_{g}$, is the set of equivalence classes of marked Riemann surfaces of genus $g$.

The Teichmüller distance between two equivalence classes $\left[X_{1}, f_{1}\right]$ and $\left[X_{2}, f_{2}\right]$ is defined as

$$
d_{T}\left(\left[X_{1}, f_{1}\right],\left[X_{2}, f_{2}\right]\right)=\frac{1}{2} \log \inf _{f} K(f)
$$

where the infimum is taken over all quasiconformal mapping $f: X_{1} \rightarrow X_{2}$ in the homotopy class of $f_{2} \circ f_{1}^{-1}$ and $K(f)$ is the quasiconformal dilation of $f$. It is well-known that the Teichmüller distance induces a complete Finsler metric on $T_{g}$. Moreover, any two points in $T_{g}$ are connected by a unique Teichmüller geodesic.

The mapping class group $\operatorname{Mod}(S)$ of $S$ is the group of isotopy classes of orientation-preserving homeomorphisms of $S$. It acts isometrically on $T_{g}$ with respect to the Teichmüller metric. There are some other natural (weak) metrics on $T_{g}$, e.g. the Thurston metric (see $\$ 2$ for definition). Unlike the Teichmüller

2010 Mathematics Subject Classification. Primary 32G15; Secondary 30F60, 60J15.

Key words and phrases. Sublinear tracking, random walks, mapping class group, Teichmüller space, Thurston's metric.

Received December 22, 2015. 
metric, the Thurston metric is asymmetric. Furthermore, geodesics connecting two points in $T_{g}$ under the Thurston metric are not unique.

The aim of this paper is to extend a theorem of Tiozzo [18] on sublinear tracking in Teichmüller distance for random walks on $\operatorname{Mod}(S)$ to the setting of the Thurston metric. Recently Walsh [20] showed that the Thurston compactification of Teichmüller space is homeomorphic to the horofunction compactification of the Thurston metric, which is useful to understanding random walks on mapping class groups $[10,6]$. This is one motivation behind our research.

1.1. Random walks on $\operatorname{Mod}(S)$. Let $\mu$ be a probability distribution on $\operatorname{Mod}(S)$, which induces a product probability distribution $\mathbf{P}:=\mu^{\mathbf{N}}$ on the sample space $\operatorname{Mod}(S)^{\mathbf{N}}($ an infinite product space of $\operatorname{Mod}(S))$. The random walk on $\operatorname{Mod}(S)$ is defined by drawing an element $g_{n}$ each time independently with probability $\mu$ from $\operatorname{Mod}(S)$ and considering the product $\omega_{n}=g_{1} g_{2} \cdots g_{n}$. If we fix a point $x$ in $T_{g}$, then $\left\{\omega_{n} x\right\}$ is a sequence of points in $T_{g}$.

We recall a few concepts that will be used later. In the following, $d(\cdot, \cdot)$ would be a metric on the Teichmüller space such that $\operatorname{Mod}(S)$ acts isometrically:

- The probability distribution $\mu$ has finite first moment if the average step is finite, that is,

$$
\int_{\operatorname{Mod}(S)} d(x, g x) d \mu(g)<\infty .
$$

- A sample path $\left\{\omega_{n} x\right\}$ has linear drift with respect to the metric $d$ if there exists a constant $A \geq 0$ such that

$$
\lim _{n \rightarrow \infty} \frac{d\left(x, \omega_{n} x\right)}{n}=A .
$$

- The random walks has sublinear tracking property if almost every sample path $\left\{\omega_{n} x\right\}$ can be approximated by some geodesic ray $\gamma:[0, \infty) \rightarrow T_{g}$ with sublinear error, i.e.

$$
\lim _{n \rightarrow \infty} \frac{d\left(\omega_{n} x, \gamma\right)}{n}=0
$$

If the distribution $\mu$ has finite first moment, it is an immediate consequence of Kingman's subadditive ergodic theorem that $\mathbf{P}$-a.e. sample path $\left\{\omega_{n} x\right\}$ has linear drift (for more details about linear drift, we refer to [9]). In [7], Kaimanovich and Masur proved that if the subgroup generated by the support of $\mu$ is non-elementary, then $\mathbf{P}$-a.e. sample path $\left\{\omega_{n} x\right\}$ converges to some point to the Thurston boundary of $T_{g}$. Recently, Tiozzo [18] solved an open question of Kaimanovich [8] by showing that random walks on $\operatorname{Mod}(S)$ has sublinear tracking property with respect to the Teichmüller metric:

THEOREM 1.1 ([18, Theorem 18]). Let $\mu$ be a probability distribution on $\operatorname{Mod}(S)$ with finite first moment with respect to the Teichmüller metric whose 
support generates a non-elementary group. Then there exists $A>0$ such that for any $x \in T_{g}$ and for $\mathbf{P}$-a.e. sample path $\left\{\omega_{n} x\right\}$ there exists a Teichmüller geodesic ray $\gamma:[0, \infty) \rightarrow T_{g}$ with $\gamma(0)=x$ and such that

$$
\lim _{n \rightarrow \infty} \frac{d_{T}\left(\omega_{n} x, \gamma(A n)\right)}{n}=0
$$

1.2. Statement of main results. In this paper, we prove that random walks on $\operatorname{Mod}(S)$ has sublinear tracking property with respect to the Thurston metric. Since the Thurston metric is asymmetric, we revise the finite first moment condition by requiring that the probability measure $\mu$ satisfies the following symmetric finite first moment property:

$$
\int_{\operatorname{Mod}(S)} \max \{d(x, g x), d(g x, x)\} d \mu(g)<\infty .
$$

THEOREM 1.2. Let $\mu$ be a distribution on $\operatorname{Mod}(S)$ with symmetric finite first moment with respect to the Thurston metric and whose support generates a nonelementary group. Then there exists $A>0$ such that for any $x \in T_{g}$ and for $\mathbf{P}$-a.e. sample path $\left\{\omega_{n} x\right\}$ there exists a stretch ray or anti-stretch ray $\gamma:[0, \infty) \rightarrow T_{g}$ such that

$$
\lim _{n \rightarrow \infty} \frac{K\left(\omega_{n} x, \gamma\right)}{n}=0 \text { and } \lim _{n \rightarrow \infty} \frac{K\left(\gamma, \omega_{n} x\right)}{n}=0
$$

where $K$ represents the Thurston metric.

Remark 1.3. 1) A stretch ray $\gamma:[0, \infty) \rightarrow T_{g}$ is a geodesic ray of the Thurston metric, a.e.

$$
K\left(\gamma\left(t_{1}\right), \gamma\left(t_{2}\right)\right)=t_{2}-t_{1}, \quad \forall 0 \leq t_{1} \leq t_{2} .
$$

We can extend $\gamma$ to a stretch line $\gamma:(-\infty, \infty) \rightarrow T_{g}$ and the anti-stretch ray $\gamma^{*}:[0, \infty) \rightarrow T_{g}$ is defined by going backward, that is,

$$
\gamma^{*}(t)=\gamma(-t), \quad t \geq 0
$$

Since the asymmetry of the Thurston metric, an anti-stretch ray may not be a geodesic ray.

2) The Teichmüller metric is an upper bound for the Thurston metric. However, they are not quasi-isometric [12]. There exist sequences $\left\{x_{n}\right\},\left\{y_{n}\right\}$ in Teichmüller space such that $d_{T}\left(x_{n}, y_{n}\right) \rightarrow \infty$ while $K\left(x_{n}, y_{n}\right) \rightarrow 0$ as $n \rightarrow \infty$, see From $[4,13]$.

Acknowledgements. I am grateful to Weixu Su and my advisor Lixin Liu for many useful discussions and corrections during the preparation of this paper. This work is partially supported by NSFC, No: 11271378 . 


\section{Preliminaries}

2.1. Thurston metric. By the Uniformization Theorem, every complex structure on $S$ is corresponding to a hyperbolic structure (a complete Riemannian metric of constant curvature -1 ) on $S$. As a result, we can define the Teichmüller space $T_{g}$ as the set of equivalence classes of marked hyperbolic structures on $S$. For simplicity, we shall always denote the equivalence class of a marked hyperbolic structure (Riemann surface) in $T_{g}$ by $x$, without explicit reference to the marking or to the equivalence relation.

Let $\mathscr{S}$ be the set of isotopy classes of simple closed curves on $S$. Endow $S$ with a hyperbolic structure, each $\alpha \in \mathscr{S}$ has a unique geodesic representation. For any $x \in T_{g}$, we choose a marked hyperbolic structure $(X, f)$ as a representation of $x$ and define $l_{x}(\alpha)$ as the length of the geodesic representation of $f(\alpha)$ on $X$. The definition of $l_{x}(\alpha)$ is independent on the choice of $(X, f)$ in the Teichmüller equivalence class and it defines a function on $T_{g} \times \mathscr{S}$.

The Thurston metric is defined as

$$
K(x, y)=\log \sup _{\alpha \in \mathscr{S}} \frac{l_{y}(\alpha)}{l_{x}(\alpha)}
$$

for any $x, y \in T_{g}$. It satisfies the following properties [19]:

- $K(x, y) \geq 0$ and $K(x, y)=0 \Leftrightarrow g=h$;

- $K(x, y) \leq K(x, z)+K(z, y)$;

- There exist $x, y \in T_{g}$ such that $K(x, y) \neq K(y, x)$.

Thus, the Thurston metric is asymmetric. Thurston [19] prove that it is Finsler and any two points in $T_{g}$ can be joined by a geodesic which is a finite concatenation of stretch line segments (but in general such a geodesic is not unique). We shall recall the construction of stretch lines in $\S 3$.

Since the Thurston metric is asymmetric, we can define a new metric $K^{*}$ as

$$
K^{*}(x, y)=K(y, x)
$$

for any $x, y \in T_{g}$. Generally, $K^{*}$ is not comparable to $K$. Choi and Rafi [4] proved that they are comparable on the thick part of $T_{g}$. In this paper, we will use the following lemma.

Lemma 2.1. Fix $x \in T_{g}$, there exists a positive constant $C$ depending on $x$ and the topology of $S$ such that

$$
K(x, y) \leq 3 K^{*}(x, y)+C
$$

for any $y \in T_{g}$.

Proof. The proof is similar to that of Proposition 4.1 in [4]. Let $l(x) \triangleq$ $\inf \left\{l_{x}(\alpha) \mid \alpha \in \mathscr{S}\right\}$. Choose a constant $\varepsilon$ which is less than $l(x)$ and the Margulis constant. Let Thick $_{\varepsilon} \triangleq\left\{z \in T_{g} \mid l_{z}(\alpha) \geq \varepsilon\right.$ for any $\left.\alpha \in \mathscr{S}\right\}$. From Theorem B in [4], there is a constant $C_{1}$ depending on $\varepsilon$ and the topology of $S$ such that

$$
\left|K(y, z)-K^{*}(y, z)\right| \leq C_{1}
$$


for any $y, z \in$ Thick $_{\varepsilon}$. Denote by $\Gamma_{y}$ the set of homotopy classes of simple closed curves whose length on $y$ is less than $\varepsilon$. It is clear that any two elements of $\Gamma_{y}$ are disjoint. Extend $\Gamma_{y}$ to a pants decomposition $\tilde{\Gamma}_{y}$ and parameterize $T_{g}$ by the Fenchel-Nielsen coordinates $\left(l\left(\alpha_{1}\right), t\left(\alpha_{1}\right), \ldots, l\left(\alpha_{3 g-3}\right), t\left(\alpha_{3 g-3}\right)\right)$, where $\alpha_{1}, \ldots, \alpha_{3 g-3} \in \tilde{\Gamma}_{y}$ (see [2] for more details about Fenchel-Nielsen coordinates). The twists parameters $t\left(\alpha_{1}\right), \ldots, t\left(\alpha_{3 g-3}\right)$ are normalized such that $t\left(\alpha_{i}\right)=1$ corresponds to the positive Dehn-twist along $\alpha_{i}$. To each $y \in T_{g}$ we associate a point $\bar{y} \in \mathrm{Thick}_{\varepsilon}$ as follows. If $y \in \mathrm{Thick}_{\varepsilon}$, then $\bar{y}=y$. If $y \in T_{g} \backslash \mathrm{Thick}_{\varepsilon}$, the Fenchel-Nielsen coordinates of $\bar{y}$ is obtained from that of $y$ by changing $l(\alpha)$ to $\varepsilon$ for any $\alpha \in \Gamma_{y}$ and keeping the others unchanged. It follows from [4, Lemma 3.3 and Theorem 3.4] that there is a constant $C_{2}$ depending on $\varepsilon$ and the topology of $S$, such that

$$
\left|\max \left\{K(y, \bar{y}), K^{*}(y, \bar{y})\right\}-\sup _{\alpha \in \Gamma_{y}}\left\{\log \frac{l_{\bar{y}}(\alpha)}{l_{y}(\alpha)}\right\}\right| \leq C_{2} .
$$

It follows that

$$
K(\bar{y}, y) \leq \sup _{\alpha \in \Gamma_{y}}\left\{\log \frac{l_{\bar{y}}(\alpha)}{l_{y}(\alpha)}\right\}+C_{2} \leq K^{*}(\bar{y}, y)+C_{2} .
$$

On the other hand, since $l(x)>\varepsilon$,

$$
\sup _{\alpha \in \Gamma_{y}}\left\{\log \frac{l_{\bar{y}}(\alpha)}{l_{y}(\alpha)}\right\}=\sup _{\alpha \in \Gamma_{y}}\left\{\log \frac{\varepsilon}{l_{y}(\alpha)}\right\} \leq K^{*}(x, y),
$$

hence

$$
K^{*}(\bar{y}, y) \leq K^{*}(x, y)+C_{2} .
$$

Combining the inequalities above, we get

$$
\begin{aligned}
K(x, y) & \leq K(x, \bar{y})+K(\bar{y}, y) \\
& \leq K^{*}(x, \bar{y})+K^{*}(\bar{y}, y)+C_{1}+C_{2} \quad(\text { by (2) and (4)) } \\
& \leq\left(K^{*}(x, y)+K^{*}(y, \bar{y})\right)+K^{*}(\bar{y}, y)+C_{1}+C_{2} \quad(\text { by (4) and (5)) } \\
& \leq 3 K^{*}(x, y)+C_{1}+3 C_{2} .
\end{aligned}
$$

Since $\varepsilon$ depends on $x$, therefore the constants $C_{1}, C_{2}$ depend on $x$ and the topology of $S$.

We need the following property of the Thurston metric due to Papadopoulos and Théret.

Proposition 2.2 ([15], Proposition 5). For any $o \in T_{q}$ and for any $R>0$, the closed balls $B_{0}(R) \triangleq\left\{y \in T_{g} \mid K(o, y) \leq R\right\}$ and $B_{o}^{*}(R) \triangleq\left\{y \in T_{g} \mid K(y, o) \leq R\right\}$, centered at $o \in T_{g}$ and of radius $R$, are compact for the usual topology. 
2.2. Measured foliations and Thurston compactification. We briefly recall the Thurston theory of measured foliations. For details we refer to [5].

A measured foliation $(F, \mu)$ on the surface $S$ is a foliation $F$ equipped with a transverse invariant measure $\mu$. More precisely, $(F, \mu)$ is determined by a finite set $\left\{Q_{i}\right\}$, called the singularities of $F$, and an atlas of coordinate charts $\left(x_{j}, y_{j}\right): U_{j} \rightarrow \mathbf{R}^{2}$ on the complement of $\left\{Q_{i}\right\}$ such that the transition functions satisfy:

$$
x_{j}=f_{k j}\left(x_{k}, y_{k}\right), \quad y_{j}= \pm y_{k}+\text { Constant }
$$

where $U_{j} \cap U_{k} \neq \emptyset$. Besides, every singularity $Q_{i}$ has $p_{i}$-prongs with $p_{i} \geq 3$. The leaves of the foliation are given by the lines $y_{i}=$ Constant and the transverse measure $\mu$ is given by $|d y|$.

The intersection number of $(F, \mu)$ with a (isotopy class of) simple closed curve $\alpha$ is defined by

$$
i(F, \mu ; \alpha)=\inf _{\alpha^{\prime}} \int_{\alpha^{\prime}}|d y|,
$$

where $\alpha^{\prime}$ is taken over all simple closed curves isotopic to $\alpha$. For simplicity, sometimes we write $F$ and $i(F, \alpha)$ instead of $(F, \mu)$ and $i(F, \mu ; \alpha)$ if there is no ambiguity about the transverse measure $\mu$.

Two measured foliations $F_{1}$ and $F_{2}$ are equivalent if $i\left(F_{1}, \alpha\right)=i\left(F_{2}, \alpha\right)$ for every $\alpha \in \mathscr{S}$. Equivalently, $F_{1}$ can be deformed into $F_{2}$ through a homeomorphism isotopic to the identity and through Whitehead moves. We denote by $\mathscr{M} \mathscr{F}(S)$ the space of equivalence classes of measured foliations on $S$. It was shown by Thurston that $\mathscr{M} \mathscr{F}(S)$ is homeomorphic to the Euclidean space of dimension $6 g-g$.

Let $\mathbf{R}_{+}^{\mathscr{S}}$ be the space of non-negative functionals on $\mathscr{S}$. We endow $\mathscr{S}$ with the discrete topology and $\mathbf{R}_{+}^{\mathscr{S}}$ with the corresponding product topology. The projective space of $\mathbf{R}_{+}^{\mathscr{S}}$ will be denoted by $\mathscr{P} \mathbf{R}_{+}^{\mathscr{S}}$. There is a natural embedding of $\mathscr{M} \mathscr{F}(S)$ into $\mathbf{R}_{+}^{\mathscr{S}}$ defined by

$$
\begin{aligned}
\mathscr{M} \mathscr{F}(S) & \rightarrow \mathbf{R}_{+}^{\mathscr{S}} \\
F & \mapsto(i(F, \alpha))_{\alpha \in \mathscr{S}} .
\end{aligned}
$$

This induces an embedding from the space of projective classes of measured foliations, denoted by $\mathscr{P} \mathscr{M} \mathscr{F}(S)$, into $\mathscr{P} \mathbf{R}_{+}^{\mathscr{S}}$. The image of $\mathscr{P} \mathscr{M} \mathscr{F}(S)$ in $\mathscr{P} \mathbf{R}_{+}^{\mathscr{S}}$ is the so called Thurston boundary, which is homeomorphic to a sphere of dimension $6 g-7$.

There is another natural embedding, from $T_{g}$ to $\mathbf{R}_{+}^{\mathscr{S}}$, given by

$$
\begin{aligned}
T_{g} & \rightarrow \mathbf{R}_{+}^{\mathscr{S}} \\
x & \mapsto\left(l_{x}(\alpha)\right)_{\alpha \in \mathscr{S}} .
\end{aligned}
$$

It was observed by Thurston that the projection of the above map to $\mathscr{P} \mathbf{R}_{+}^{\mathscr{S}}$ is also an embedding. Moreover, the closure of the image of $T_{g}$ in $\mathscr{P} \mathbf{R}_{+}^{\mathscr{S}}$ is a compact 
set, called the Thurston compactification of $T_{g}$. The boundary of the Thurston compactification is the Thurston boundary, which, as we mentioned above, can be identified with the space of projective measured foliations $\mathscr{P} \mathscr{M} \mathscr{F}(S)$.

A measured foliation $(F, \mu)$ is minimal if $i(F, \mu ; \alpha)>0$ for every simple closed curve $\alpha . \quad(F, \mu)$ is uniquely ergodic if the transverse measure $\mu$ admitted on the underlying foliation $F$ is unique up to a positive scalar.

2.3. Measured laminations. Let us endow $S$ with a hyperbolic structure $m_{0}$. A geodesic lamination on $S$ is a closed subset of $S$ consisting of disjoint union of complete simple geodesics (called the leaves) on $S$. Each leaf is either a simple closed geodesic or a bi-infinite geodesic. Denote by $\mathscr{L}_{m_{0}}(S)$ the space of all geodesic laminations on $S$. Since geodesic laminations are closed subsets of $S$, we use the Hausdorff distance to define the topology on $\mathscr{L}(S)$.

Proposition 2.3 (see [1]). The space $\mathscr{L}_{m_{0}}(S)$ with the Hausdorff distance is compact.

It turns out that the notion of geodesic lamination is independent on the choice of hyperbolic structure on $S$. For two different choice of hyperbolic metrics $m_{0}$ and $m_{1}$, there is a natural homeomorphism between $\mathscr{L}_{m_{0}}(S)$ and $\mathscr{L}_{m_{1}}(S)$. We can use $\mathscr{L}(S)$ to represent the space of geodesic laminations on $S$ without reference to any particular hyperbolic metric on $S$.

A measured geodesic lamination is a geodesic lamination $L$ equipped with a full support transverse invariant measure $\mu$, which means that if $a$ and $b$ are arcs transverse to $L$ and are homotopic through transverse arcs with endpoints not contained in $L$ then $\mu(a)=\mu(b)$. The intersection number $i(\mu, \alpha)$ of $\mu$ with a simple closed curve $\alpha$ is defined in the same way as that of a measured foliation. As a consequence, every measured geodesic lamination can be viewed as a functional on the space of isotopy classes of simple closed curves. So the space of measured geodesic laminations $\mathscr{M} \mathscr{L}(S)$ can also be embedded into $\mathbf{R}_{+}^{\mathscr{S}}$. In fact, there is one-to-one correspondence between $\mathscr{M} \mathscr{F}(S)$ and $\mathscr{M} \mathscr{L}(S)$ as described by Levitt in [11].

Theorem 2.4 ([11], Theorem 1, 2). Let X be a closed orientable hyperbolic surface.

A foliation $F$ on $X$ is canonically associated with a geodesic lamination $\gamma(F)$ and a family $e(F)$ of leaves of this lamination; this family is at most countably infinite and contains all isolated leaves of $\gamma(F)$. If $F$ and $F^{\prime}$ are topologically equivalent foliations, then $\gamma(F)=\gamma\left(F^{\prime}\right)$ and $e(F)=e\left(F^{\prime}\right)$.

Given a lamination $\gamma$ and a family e of leaves of $\gamma$ as above, there exists a foliation $F$ such that $\gamma(F)=\gamma$ and $e(F)=e$. This foliation is unique up to topological equivalence.

Given a transverse measure $\mu$ of $F$, there exists a unique transverse measure $\mu^{\prime}$ of $\gamma(F)$ satisfying $i(F, \mu ; \alpha)=i\left(\gamma(F), \mu^{\prime} ; \alpha\right)$ for every simple closed curve $\alpha$ on $S$. 
Hence, the space of projective measured geodesic laminations $\mathscr{P} \mathscr{M} \mathscr{L}(S)$ can also be viewed as the Thurston's boundary of $T_{g}$.

Remark 2.5. There are geodesic laminations which do not admit a full support transverse invariant measure. In fact, a geodesic lamination admits a full support transverse invariant measure if and only if it contains no isolated leaves. Here an isolated leaf $\alpha$ of a lamination $L$ means that for each $x \in \alpha$ there exists a neighbourhood $O$ of $x$ such that $O \cap L$ has only one connected component.

Definition (Measure topology on $\mathscr{M} \mathscr{L}(S)$ and $\mathscr{M} \mathscr{F}(S))$. Let $\left\{\mu_{k}\right\}_{k=0}^{\infty}$ be a sequence in $\mathscr{M} \mathscr{L}(S)$ (resp. $\mathscr{M} \mathscr{F}(S)$, we say $\mu_{k}$ converges to $\mu$ in the measure topology if for any simple closed curve $\alpha, i\left(\mu_{k}, \alpha\right)$ converges to $i(\mu, \alpha)$ as $k$ tends to infinity.

\section{Some properties of Teichmüller space with the Thurston metric}

3.1. Stretch lines. We sketch the construction of a "stretch line" which is a special kind of geodesic line under the Thurston metric. Let $h$ be a hyperbolic structure on $S$ and $\mu$ a complete geodesic lamination on $S$ (a complete geodesic lamination is a lamination whose complement on $S$ consists of ideal triangles). We define a L-Lipschitz homeomorphism for every ideal triangle. Each corner of an ideal triangle can be foliated by horocycles. Extending these foliations until they fill all but region in the center bounded by three horocycles (see Figure 1(a)). The L-Lipschitz homeomorphism can be constructed by fixing the central

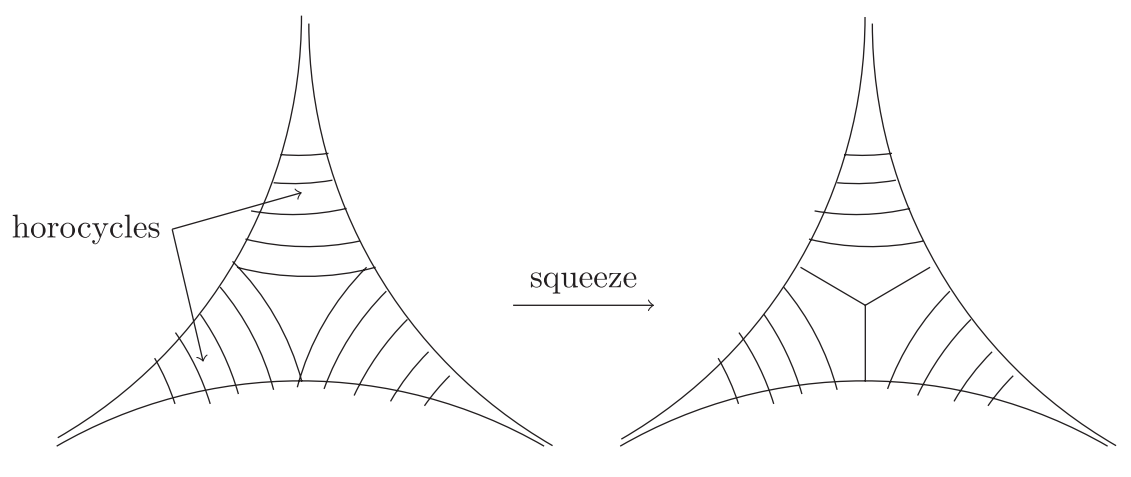

(a)

(b)

FIGURE 1. Horocyclic foliation on an ideal triangle. In figure (a) the horocycles foliate the ideal triangle except the center region; in figure (b), the center region has been squeezed to a "Y" piece and the desired foliation is obtained. 
region, and mapping a horocycle which has distance $t$ from the central region to the horocycle with distance $L t$. These L-Lipschitz homeomorphisms defines a $L$-Lipschitz homeomorphism $f(h, \mu, L)$ of $S$ to itself, and hence a new hyperbolic structure $h^{\prime}$ which is the push-forward of $g$ by $f(h, \mu, L)$. Sometimes, the new hyperbolic structure $h^{\prime}$ may be equivalent to $h$. For example, a L-Lipschitz homeomorphism on an ideal triangle does not change the hyperbolic structure on it because all the hyperbolic structures on an ideal triangle are isometric. But if $S$ is a closed surface with genus $g \geq 2$, which is the case we considered here, $h^{\prime}$ is different from $h$. Furthermore, the Thurston distance is $K\left(h, h^{\prime}\right)=\log L$. Let $L$ vary among positive numbers, we get a line $\operatorname{stretch}(h, \mu, L)$ in the Teichmüller space $T_{g}$. It is a geodesic line under the Thurston metric and we call it a "stretch line".

3.2. Cataclysm coordinate system. In [19], Thurston introduced a cataclysm coordinate system for Teichmüller space.

Fix a hyperbolic structure $m$ on the surface $S$. For any complete geodesic lamination $\mu$, we denote by $\mathscr{M} \mathscr{F}(\mu)$ the set of measured foliation (class) that are transverse to $\mu$. Here a measured foliation $F$ is transverse to $\mu$ means that no disk can be bounded by two arcs such that one is from a leaf of $F$ and the other is from a leaf of $\mu$. As we describe in Section 3.1, the complement of the center region of an ideal triangle can be foliated by horocycles. If we squeeze the horocycles with the endpoints in $\mu$ unmoved such that the center region becomes a "Y" piece (see Figure 1(b)), then we get a foliation on each ideal triangle and hence a foliation $F_{\mu}(m)$ on the surface $S$. Furthermore, we can associate a transverse measure to $F_{\mu}(m)$ by identifying $i\left(F_{\mu}(m), \gamma\right)$ with the length of $\gamma$ under the hyperbolic metric $m$ for any arc $\gamma$ contained in a leaf of $\mu$. So we get a measured foliation (still denoted as $\left.F_{\mu}(m)\right)$ on $S$, which we call horocyclic measured foliation associated to $\mu$. If $h$ is a hyperbolic metric which is equivalent to $m$, then the horocyclic measured foliation $F_{\mu}(h)$ is isotopic to $F_{\mu}(m)$. This enables us to associate to each $m$ in $T_{g}$ a well-defined element in $\mathscr{M} \mathscr{F}(\mu)$. We denote by $\phi_{\mu}: T_{g} \rightarrow \mathscr{M} \mathscr{F}(\mu)$ the resulting map.

THEOREM 3.1 ([19], §9). For any complete geodesic lamination $\mu$ on $S$, the map $\phi_{\mu}: T_{g} \rightarrow \mathscr{M} \mathscr{F}(\mu)$ is a homeomorphism.

The global coordinates for Teichmüller space that are provided by this map $\phi_{\mu}$ are called by Thurston cataclysm coordinates.

Now we consider a special kind of complete geodesic lamination, that is, chain recurrent lamination. A lamination $\mu$ is called chain recurrent if for any $\varepsilon$ and for any $x \in \mu$ there is a closed $\varepsilon$-trajectory of $\mu$ through $x$, that is, a closed unit speed path in the surface such that for any interval of length 1 on the path there is an interval of length 1 on some leaf of $\mu$ such that the two paths remain within $\varepsilon$ of each other in the $C^{1}$ sense. Any measured lamination is chain recurrent ([19], [16]). Denote by $\mathscr{C} \mathscr{L}(S)$ the set of complete, chain recurrent geodesic laminations on $S$. We have the following Theorem. 
TheOREM 3.2 (Cataclysm coordinate system [19], §10). Let $U \subset \mathscr{C} \mathscr{L}(S) \times$ $\mathscr{M} \mathscr{F}(S)$ be a set consisting of pairs $(\mu, F)$ where $F$ is a measured foliation transverse to $\mu$. If we equip $U$ with the product topology of the Hausdorff topology on $\mathscr{C} \mathscr{L}(S)$ and the weak topology on $\mathscr{M} \mathscr{F}(S)$. Then there exists a continuous map $c: U \rightarrow T_{g}$.

So for a given pair $(\mu, F) \in U$, we have a stretch line $\gamma_{\mu, F}$,

$$
\gamma_{\mu, F}(t) \triangleq \operatorname{stretch}(c(\mu, F), \mu, \exp (t))
$$

directed by $\mu$ and starting at $c(\mu, F)$. Moreover, the stretch line $\gamma_{\mu, s F}$ is the same as $\gamma_{\mu, F}$ after reparametrization (for more details about cataclysm coordinate system and stretch lines we refer to [19]).

3.3. Two auxiliary functions. Fix a point $o \in T_{g}$, we define a function $D$ on $U$ as

$$
\begin{aligned}
D: \quad U & \rightarrow \mathbf{R}^{+} \\
(\mu, F) & \mapsto K\left(\gamma_{\mu, F}, o\right)=\inf _{t \in \mathbf{R}} K\left(\gamma_{\mu, F}(t), o\right)
\end{aligned}
$$

where $K($,$) is the Thurston metric.$

Proposition 3.3. D is continuous.

Proof. Fix a point $m \in T_{q}$, we have a hyperbolic structure on $S$, then $\mathscr{C} \mathscr{L}(S)$ has a Hausdorff metric (This is the only situation we need $m$ ). Let $\left(\mu_{n}, F_{n}\right) \in U$ with $\left(\mu_{n}, F_{n}\right) \rightarrow\left(\mu_{0}, F_{0}\right)$, as $n \rightarrow \infty$. We need to show $\lim _{n \rightarrow \infty} D\left(\mu_{n}, F_{n}\right)=$ $D\left(\mu_{0}, F_{0}\right)$.

Pick the point $x_{0}=r_{\mu_{0}, F_{0}}\left(t_{0}\right) \in r_{\mu_{0}, F_{0}}$ which realizes $D\left(\mu_{0}, F_{0}\right)$. So $x_{0}$ corresponds to $c\left(\mu, \exp \left(t_{0}\right) F\right)$ in the cataclysm coordinates. Choose the points $x_{n}=$ $c\left(\mu_{n}, \exp \left(t_{0}\right) F_{n}\right) \in r_{\mu_{n}, F_{n}}$. We get

$$
\begin{gathered}
\lim _{n \rightarrow \infty} x_{n}=x_{0}, \\
\limsup _{n \rightarrow \infty} D\left(\mu_{n}, F_{n}\right) \leq \lim _{n \rightarrow \infty} K\left(x_{n}, o\right)=K\left(x_{0}, o\right)=D\left(\mu_{0}, F_{0}\right) .
\end{gathered}
$$

To prove the opposite direction, we choose a subsequence $y_{k_{n}}=c\left(\mu_{k_{n}}, \exp \left(t_{k_{n}}\right) F_{k_{n}}\right)$ $\in r_{\mu_{k_{n}}, F_{k_{n}}}$ realizing $D\left(\mu_{k_{n}}, F_{k_{n}}\right)$, so that

$$
\lim _{n \rightarrow \infty} K\left(y_{k_{n}}, o\right)=\liminf _{n \rightarrow \infty} D\left(\mu_{n}, F_{n}\right) .
$$

It follows from Proposition 2.2 that $\left\{y_{k_{n}}\right\}$ stays in a compact subset of $T_{g}$. Then there is a subsequence of $\left\{t_{k_{n}}\right\}$ converging to some positive number $t_{k_{0}}$. For simplicity we still denote the subsequence as $\left\{t_{k_{n}}\right\}$. Hence

$$
\lim _{n \rightarrow \infty} y_{k_{n}}=c\left(\mu_{0}, \exp \left(t_{k_{0}}\right) F_{0}\right) \triangleq y_{0} \in \gamma_{\mu_{0}, F_{0}},
$$

where we use the fact $\lim _{n \rightarrow \infty}\left(\mu_{n}, F_{n}\right)=\left(\mu_{0}, F_{0}\right)$. 
Then, we get the opposite inequality

$$
D\left(\mu_{0}, F_{0}\right) \leq K\left(y_{0}, o\right)=\lim _{n \rightarrow \infty} K\left(y_{k_{n}}, o\right)=\liminf _{n \rightarrow \infty} D\left(\mu_{n}, F_{n}\right) .
$$

By the one-to-one correspondence between $\mathscr{M} \mathscr{F}(S)$ and $\mathscr{M} \mathscr{L}(S)$, we can replace the domain $U$ of the map $D$ by $U^{\prime}(\subset \mathscr{C} \mathscr{L}(S) \times \mathscr{M} \mathscr{L}(S))$ consisting of transverse pairs $(\mu, \lambda)$ where the corresponding measured foliation $F(\lambda)$ of $\lambda$ defined by Theorem 2.4 is transverse to $\mu$. For simplicity, we still denote the function from $U^{\prime}$ to $\mathbf{R}^{+}$as $D$.

Next, we need to modify $D$ a little bit. For a measured lamination $\xi$, let $W(\xi)$ be the set of complete, chain recurrent geodesic laminations containing $\xi$. By Theorem 1.3.6 in [16], we know that $W(\xi)$ is not empty for any measured foliation $\xi$. Since the completion only relates to the underlying lamination, $W(\xi)=W(s \xi)$ for any positive number $s$. For convenience, denote by $W([\xi]) \triangleq W(\xi)$, where $[\xi]$ represents the projective class of $\xi$.

Recall that a measured geodesic lamination $\mu$ is called uniquely ergodic if the transverse measure admitted on the underlying geodesic lamination $L$ is unique up to multiplication by a positive constant. $\mu$ is said to fill up the surface $S$ if $i(\mu, \alpha)>0$ for any simple closed curve $\alpha$ on $S$, or equivalently, the complementary of the underlying geodesic lamination $L$ of $\mu$ on $S$ consists of several simply connected regions. Let $\mathscr{M} \mathscr{L} \mathscr{N}(S) \subset \mathscr{P} \mathscr{M} \mathscr{L}(S)$ consist of measured geodesic laminations which is uniquely ergodic and fills up the surface $S$. For any $\xi \in \mathscr{M} \mathscr{L} \cdot \mathscr{N}(S)$, we can easily get that if $F$ is a measured foliation transverse to $\xi$ and $\mu$ is an element of $W([\xi])$, then $F$ is also transverse to $\mu$. Let $V \subset \mathscr{M} \mathscr{L} \mathscr{N}(S) \times \mathscr{M} \mathscr{L} \mathscr{N}(S)$ consist of transverse pairs $([\xi],[\eta])$. We can define a map $P$ from $V$ to a set $\mathscr{P}\left(\Gamma T_{g}\right)$ consisting of collections of stretch lines as below:

$$
\begin{aligned}
& P: \quad V \rightarrow \mathscr{P}\left(T_{g}\right) \\
& ([\xi],[\eta]) \mapsto\left\{\gamma_{\mu, \eta}: \mu \in U([\xi])\right\} .
\end{aligned}
$$

The mapping class $\operatorname{group} \operatorname{Mod}(S)$ acts naturally on $\mathscr{P} \mathscr{M} \mathscr{L}(S)$ and the intersection number is $\operatorname{Mod}(S)$-invariant, i.e.,

$$
i\left(g \xi_{1}, g \xi_{2}\right)=i\left(\xi_{1}, \xi_{2}\right) \quad \forall g \in \operatorname{Mod}(S), \quad \xi_{1}, \xi_{2} \in \mathscr{P} \mathscr{M} \mathscr{L}(S) .
$$

Besides, the mapping class group $\operatorname{Mod}(S)$ acts isometrically on Teichmüller space $T_{g}$ with respect to the Thurston metric, so the map $P$ is $\operatorname{Mod}(S)$-equivariant, i.e.,

$$
P([g \xi],[g \eta])=g P([\xi],[\eta]) \triangleq\left\{g\left(\gamma_{\mu, \eta}\right): \mu \in W([\xi])\right\},
$$

where $g \in \operatorname{Mod}(S)$ and $([\xi],[\eta]) \in V$.

Next we define a function $D_{0}$ on $V$ by:

$$
\begin{aligned}
& D_{0}: \quad V \rightarrow \mathbf{R}^{+} \\
& ([\xi],[\eta]) \mapsto \inf _{\gamma \in \mathscr{P}([\xi],[\eta])} K(\gamma, o)=\inf _{\mu \in w([\xi])} D(\mu, \eta) .
\end{aligned}
$$


Lemma 3.4. There is a stretch line $\gamma \in \mathscr{P}([\xi],[\eta])$ such that $D_{0}([\xi],[\eta])=$ $K(o, \gamma)$.

Proof. There is a sequence $\mu_{n} \in W([\xi])$ such that

$$
D_{0}([\xi],[\eta])=\lim _{n \rightarrow \infty} K\left(o, \gamma_{\mu_{n}, \eta}\right)=\lim _{n \rightarrow \infty} D\left(\mu_{n}, \eta\right) .
$$

By Proposition 2.3 , the space $\mathscr{L}_{m_{0}}(S)$ of geodesic laminations is compact in the Hausdorff topology. There is subsequence $\left\{\mu_{n_{k}}\right\}_{k=1}^{\infty}$ of $\left\{\mu_{n}\right\}_{n=1}^{\infty}$ such that $\mu_{n_{k}}$ converges to some geodesic lamination $\mu$ in the Hausdorff topology. It is clear that $\mu$ is also a complete geodesic lamination containing the underlying geodesic lamination of $\xi$, therefore $\xi \in W([\xi])$. From Proposition 3.3, it follows

$$
\lim _{k \rightarrow \infty} D\left(\mu_{n_{k}}, \eta\right)=D(\mu, \eta)=K\left(\gamma_{\mu, \eta}, o\right) .
$$

Proposition 3.5. $D_{0}$ is lower semi-continuous and Borel measurable.

Before we prove Proposition 3.5, we need some preparations. For a measured lamination $\lambda$, there is a canonically associated set $C(\lambda)$ of geodesic laminations, consisting of the intersection of the closures in the Hausdorff topology of its neighbourhoods in the measure topology. $C(\lambda)$ consists of only $\lambda$ itself if and only if $\lambda$ is complete. In [19], Thurston proved the following propositions.

Proposition 3.6 (Chain recurrence is closed, [19] Proposition 6.2). Any compactly-supported lamination which is a Hausdorff limit of a chain recurrent laminations is chain recurrent.

Proposition 3.7 (Hausdorff closure of neighbourhoods, [19] Proposition 6.3). $C(\lambda)$ consists of all chain recurrent laminations which contains $\lambda$.

Lemma 3.8. Assume $\eta_{n} \in \mathscr{M} \mathscr{L}(S), n=1,2 \ldots$ and $\eta_{n}$ converges to $\eta_{0}$ in the measure topology as $n$ tends to infinity. Let $\mu_{n} \in W\left(\left[\eta_{n}\right]\right)$, then the limit points of $\left\{\mu_{n}\right\}$ in the Hausdorff topology are contained in $W\left(\left[\eta_{0}\right]\right)$.

Proof. Let $\mu^{\prime}$ be a limit point of $\left\{\mu_{n}\right\}_{n=1}^{\infty}$ in the Hausdorff topology. There exists a subsequence $\left\{\mu_{n_{k}}\right\}$ converges to $\mu^{\prime}$ in the Haudorff topology. By Proposition 3.6, $\mu^{\prime}$ is a complete chain recurrent geodesic lamination.

On the other hand, $\eta_{n_{k}}$ converges to $\eta_{0}$ in the measure topology as $n$ tends to infinity. From Proposition 3.7 and Theorem 2.3, we know that there exists a subsequence of $\eta_{n_{k}}$, still denoted as $\eta_{n_{k}}$ for simplicity, converges to a chain recurrent lamination $\eta^{\prime} \in C\left(\eta_{0}\right)$ in the Hausdorff topology.

Therefore, $\eta^{\prime} \subset \mu^{\prime}$, which means that $\mu^{\prime}$ is a completion of $\eta^{\prime}$ and hence a completion of $\eta_{0}$. 
After the preparations above, we prove Proposition 3.5 now.

Proof of Proposition 3.5. Let $\left(\left[\eta_{n}\right],\left[\xi_{n}\right]\right) \in V$ with $\left(\left[\eta_{n}\right],\left[\xi_{n}\right]\right)$ converges to $\left(\left[\eta_{0}\right],\left[\xi_{0}\right]\right)$ as $n$ tends to infinity. There exists $s_{n}, r_{n} \geq 0$ such that

$$
\begin{aligned}
& s_{n} \eta_{n} \rightarrow \eta_{0}, \quad \text { in measure topology, } \\
& r_{n} \xi_{n} \rightarrow \xi_{0}, \quad \text { in measure topology, }
\end{aligned}
$$

as $n \rightarrow \infty$. Take a subsequence $\left\{\left(s_{n_{k}} \eta_{n_{k}}, r_{n_{k}} \xi_{n_{k}}\right)\right\}$ such that

$$
\lim _{k \rightarrow \infty} D_{0}\left(\left[\eta_{n_{k}}\right],\left[\xi_{n_{k}}\right]\right)=\liminf _{n \rightarrow \infty} D_{0}\left(\left[\eta_{n}\right],\left[\xi_{n}\right]\right) .
$$

It follows from Lemma 3.4 that there exist $x_{n_{k}} \in T_{g}, t_{n_{k}} \in \mathbf{R}$, and $\mu_{n_{k}} \in W\left(s_{n_{k}} \eta_{n_{k}}\right)$ $=W\left(\eta_{n_{k}}\right)$ such that $x_{n_{k}}=\gamma_{\mu_{n_{k}}, r_{n_{k}} \xi_{n_{k}}}\left(t_{n_{k}}\right)$ realizes $D_{0}\left(\left[\eta_{n_{k}}\right],\left[\xi_{n_{k}}\right]\right)$, i.e. $K\left(x_{n_{k}}, o\right)=$ $D_{0}\left(\left[\eta_{n_{k}}\right],\left[\xi_{n_{k}}\right]\right)$. By Theorem 2.3 and Lemma 3.8, there is a subsequence of $\left\{n_{k}\right\}_{k=1}^{\infty}$, which is still denoted as $\left\{n_{k}\right\}_{k=1}^{\infty}$ for simplicity, such that $\mu_{n_{k}} \rightarrow \mu_{0}$ for some $\mu_{0} \in W\left(\left[\eta_{0}\right]\right)$ as $k \rightarrow \infty$.

We claim that $\left\{t_{n_{k}}\right\}$ is bounded. In fact,

$$
\begin{aligned}
t_{n_{k}} & =K\left(\gamma_{\mu_{n_{k}}, r_{n_{k}} \xi_{n_{k}}}(0), \gamma_{\mu_{n_{k}}, r_{n_{k}} \xi_{n_{k}}}\left(t_{n_{k}}\right)\right) \\
& \leq K\left(\gamma_{\mu_{n_{k}}, r_{n_{k}} \xi_{n_{k}}}(0), o\right)+K\left(o, \gamma_{\mu_{n_{k}}, r_{n_{k}} \xi_{n_{k}}}\left(t_{n_{k}}\right)\right) .
\end{aligned}
$$

It follows from Theorem 3.2 that $K\left(\gamma_{\mu_{n_{k}}, r_{n_{k}} \xi_{n_{k}}}(0), o\right)$ is bounded. On the other hand, by (6),

$$
\lim _{k \rightarrow \infty} D_{0}\left(\left[\eta_{n_{k}}\right],\left[\xi_{n_{k}}\right]\right)=\lim _{k \rightarrow \infty} K\left(\gamma_{\mu_{n_{k}}, r_{n_{k}} \xi_{n_{k}}}\left(t_{n_{k}}\right), o\right) .
$$

By Proposition 2.2, the sequence $\left\{\gamma_{\mu_{n_{k}},}, r_{n_{k}} \xi_{n_{k}}\left(t_{n_{k}}\right)\right\}_{k=1}^{\infty}$ stays in a compact subset of $T_{g}$, which means $K\left(o, \gamma_{\mu_{n_{k}}}, r_{n_{k}} \xi_{n_{k}}\left(t_{n_{k}}\right)\right)$ is bounded. Hence, $\left\{t_{n_{k}}\right\}$ is bounded.

There is a subsequence of $\left\{n_{k}\right\}_{k=1}^{\infty}$, which is still denoted as $\left\{n_{k}\right\}_{k=1}^{\infty}$ for simplicity, such that $t_{n_{k}}$ converges to some $t_{0} \in \mathbf{R}$ as $k \rightarrow \infty$.

As a consequence,

$$
\lim _{k \rightarrow \infty} x_{n_{k}}=\gamma_{\mu_{0}, \xi_{0}}\left(t_{0}\right) \triangleq x_{0}
$$

Hence

$$
D_{0}([\eta],[\xi]) \leq K\left(x_{0}, o\right)=\lim _{k \rightarrow \infty} K\left(x_{n_{k}}, o\right)=\liminf _{k \rightarrow \infty} D_{0}\left(\left[\eta_{n}\right],\left[\xi_{n}\right]\right) .
$$

So $D_{0}$ is lower semi-continuous and hence Borel measurable.

Recall that the stump of a geodesic lamination $\mu$ is the maximal sublamination of $\mu$ admitting a transverse measure. The stretch line $\gamma_{\mu, \eta}(t)$ converges to the projective class $[\eta]$ of $\eta$ in $\mathscr{P} \mathscr{M L}(S)$ in the positive direction ([14]). But the convergence of $\gamma_{\mu, \eta}(t)$ in the negative direction does not alway exist. Neverthe- 
less, if the stump of $\mu$ is uniquely ergodic, $\gamma_{\mu, \eta}(t)$ converges to the projective class of the stump in $\mathscr{P} \mathscr{M} \mathscr{L}(S)$ in the negative direction ([17] $\$ 3.3$ Theorem 7).

Proposition 3.9. Let $([\xi],[\eta]) \in V$. Then

1. any stretch line $\gamma_{\mu, \eta}(t) \in P([\xi],[\eta])$ converges to $[\eta]$ in the positive direction and converges to $[\xi]$ in the negative direction;

2. any two stretch lines in $P([\xi],[\eta])$ are kept in bounded distance.

Proof. For the first part, recall that $\xi$ is uniquely ergodic and fills up the surface $S$. Then the complementary region of $\xi$ on the surface $S$ are ideal polygons, which means that every completion $\mu$ of $\xi$ is the union of $\xi$ with finite isolated, infinite leaves. Since the support of a measured lamination contains no isolated infinite leaves, the maximal measured lamination contained by $\mu$ is $\xi$, which is to say that $\xi$ is the stump of $\mu$. Then the stretch line $\gamma_{\mu, \eta}(t)$ converges to $[\xi]$ in the negative direction $([17] \S 3.3$ Theorem 7) and converges to $[\eta]$ in the positive direction ([14]).

The second part of the conclusion follows from Proposition 3.10 and the first part we just prove.

Proposition 3.10 ([17] \$3.6 Proposition 4). Given two measured geodesic laminations $\lambda, \gamma$ meeting every component of one another and such that $\gamma$ is uniquely ergodic, we can find a stretch line having $[\lambda]$ as positive endpoint and $[\gamma]$ as negative endpoint. This stretch line is unique if and only if $[\gamma]$ is complete. In any ways, the distance between two such stretch lines is bounded from above.

\section{Proof of Theorem $\mathbf{1 . 2}$}

In this section, we follow Tiozzo's idea [18] to prove Theorem 1.2. First of all, we collect some results which will be useful in our proof.

The first one is a technical lemma whose proof can be found in [18].

Lemma 4.1 ([18], Lemma 7). Let $\Omega$ be a measure space with a probability measure $\lambda$, and let $T: \Omega \rightarrow \Omega$ be a measure-preserving, ergodic transformation. Let $f: \Omega \rightarrow \mathbf{R}$ be a non-negative, measurable function, and define the function $g: \Omega \rightarrow \mathbf{R}$ as

If $g \in L^{1}(\Omega, \lambda)$, then, for $\lambda$-almost every $\omega \in \Omega$,

$$
g(\omega):=f(T \omega)-f(\omega), \quad \forall \omega \in \Omega,
$$

$$
\lim _{n \rightarrow \infty} \frac{f\left(T^{n} \omega\right)}{n}=0
$$

The second one concerns the linear drift of the random walk. The result is a little bit different from the original one, but both of them can be obtained from Kingman's subadditive ergodic theorem. 
Proposition 4.2 ([18], Proposition 4). Let $\mu$ be a distribution on $\operatorname{Mod}(S)$ with symmetric finite first moment, then there is $A>0$ such that for each $x \in T_{g}$ and for $\mathbf{P}$ a.e. sample path

$$
\lim _{n \rightarrow \infty} \frac{K\left(\omega_{n} x, x\right)}{n}=A
$$

Remark 4.3. The constant $A$ is only nonnegative in the original statement in [18] under more general settings. The positivity in this particular setting is proved in the proof of Theorem 18 from [18].

The last one is about the convergence sample paths in $T_{g}$.

THEOREM 4.4 ([7], Theorem 2.2.4). Let $\mu$ be a probability measure on $\operatorname{Mod}(S)$ such that the group generated by the support of $\mu$ is non-elementary. For P-a.e. sample path $\omega=\left\{\omega_{n}\right\}$ of the random walk and any $x \in T_{g}$ the sequence $\omega_{n} x$ converges in the Thurston boundary $\mathscr{P} \mathscr{M} \mathscr{F}$ to an uniquely ergodic measured foliation $F(\omega)$.

Now, we prove Theorem 1.2.

Proof of Theorem 1.2. The proof will be split into two steps. In step 1, we will show that for $\mathbf{P}$-a.e. sample path $\left\{\omega_{n} x\right\}_{n=1}^{\infty}$, there is a stretch line $\gamma:(-\infty,+\infty) \rightarrow T_{g}$ such that

$$
\lim _{n \rightarrow \infty} \frac{\max \left\{K\left(\omega_{n} x, \gamma\right), K\left(\gamma, \omega_{n} x\right)\right\}}{n}=0 .
$$

Then, in step 2, we will show the stretch line $\gamma$ chosen in step 1 can be replaced by a stretch ray or anti-stretch ray.

STEP 1. Set $\Omega=\operatorname{Mod}(S)^{\mathbf{Z}}$ be the product space of $\operatorname{Mod}(S)$. Let $T$ be the left shift of $\Omega$. That is to say, if we let $a_{n}(g) \triangleq g_{n}$ for $g \in \Omega$, then $a_{n}(T g)=g_{n+1}$.

By Theorem 4.4, to P-a.e. sample path $g=\left\{\omega_{n} x\right\}$ we can associate two points bnd $^{+}(g)$, bnd ${ }^{-}(g) \in \partial X$ as below:

$$
\begin{gathered}
\text { bnd }^{+}(g) \triangleq \lim _{n \rightarrow \infty} \omega_{n} x=\lim _{n \rightarrow \infty} g_{1} \cdots g_{n} x . \\
\text { bnd }^{-}(g) \triangleq \lim _{n \rightarrow \infty} \bar{\omega}_{n} x=\lim _{n \rightarrow \infty} g_{0}^{-1} g_{-1}^{-1} \cdots g_{-n}^{-1} x .
\end{gathered}
$$

Then we have

$$
\text { bnd }^{+}\left(T^{m} g\right)=\lim _{n \rightarrow \infty} T^{m} \omega_{n} x=\lim _{n \rightarrow \infty} \omega_{m}^{-1} g_{1} \cdots g_{n} x=\omega_{m}^{-1} \text { bnd }^{+}(g),
$$

and

$$
\text { bnd }^{-}\left(T^{m} g\right)=\omega_{m}^{-1} \text { bnd }^{-}(g)
$$


By Proposition 3.5, we know the function

$$
f(g) \triangleq D_{0}\left(\text { bnd }^{-}(g), \text { bnd }^{+}(g)\right)=\inf _{\gamma \in P\left(\text { bnd }^{-}(g), \text { bnd }^{+}(g)\right)} K(\gamma, x)
$$

is measurable and finite $\mathbf{P}$-a.e.. Further,

$$
\begin{aligned}
f\left(T^{m} g\right) & =\inf _{\gamma \in P\left(\mathbf{b n d}^{-}\left(T^{m} g\right), \text { bnd }^{+}\left(T^{m} g\right)\right)} K(\gamma, x) \\
& =\inf _{\gamma \in P\left(\omega_{m}^{-1} \mathbf{b n d}^{-}(g), \omega_{m}^{-1} \mathbf{b n d}^{+}(g)\right)} K(\gamma, x) \\
& =\inf _{\omega_{m} \gamma \in P\left(\mathbf{b n d}^{-}(g), \text { bnd }^{+}(g)\right)} K\left(\omega_{m} \gamma, \omega_{m} x\right) \\
& =\inf _{\gamma \in P\left(\mathbf{b n d}^{-}(g), \text { bnd }^{+}(g)\right)} K\left(\gamma, \omega_{m} x\right) .
\end{aligned}
$$

And

$$
\begin{aligned}
|f(T g)-f(g)| & =\left|\inf _{\gamma \in P\left(\mathbf{b n d}^{-}(g), \text { bnd }^{+}(g)\right)} K\left(\gamma, \omega_{1} x\right)-\inf _{\gamma \in P\left(\mathbf{b n d}^{-}(g), \text { nnd }^{+}(g)\right)} K(\gamma, x)\right| \\
& \leq \max \left\{K\left(g_{1} x, x\right), K\left(x, g_{1} x\right)\right\} .
\end{aligned}
$$

Hence $|f(T g)-f(g)|$ is integrable because of the assumption that $\mu$ has symmetric finite first moment. Then, we get, by Lemma 4.1,

$$
\lim _{n \rightarrow \infty} \frac{f\left(T^{n} g\right)}{n}=\lim _{n \rightarrow \infty} \inf _{\gamma \in P\left(\mathbf{b n d}^{-}(g), \text { bnd }^{+}(g)\right)} \frac{K\left(\gamma, \omega_{n} x\right)}{n}=0,
$$

so there exists $\gamma_{n} \in P\left(\right.$ bnd $^{-}(g)$, bnd $\left.{ }^{+}(g)\right)$ for each $n$ such that

$$
\lim _{n \rightarrow \infty} \frac{K\left(\gamma_{n}, \omega_{n} x\right)}{n}=0 .
$$

By Proposition 3.10, any two stretch lines in $P\left(\mathbf{b n d}^{-}(g)\right.$, bnd $\left.^{+}(g)\right)$ are kept in bounded distance $C$, hence

$$
\lim _{n \rightarrow \infty} \frac{K\left(\gamma, \omega_{n} x\right)}{n} \leq \lim _{n \rightarrow \infty} \frac{K\left(\gamma_{n}, \omega_{n} x\right)+C}{n}=0, \quad \forall \gamma \in P\left(\text { bnd }^{-}(g), \text { bnd }^{+}(g)\right) .
$$

Combining with Lemma 2.1, we get

$$
\begin{aligned}
\lim _{n \rightarrow \infty} \frac{K\left(\omega_{n} x, \gamma\right)}{n} & =\lim _{n \rightarrow \infty} \frac{K\left(x, \omega_{n}^{-1} \gamma\right)}{n} \\
& \leq \lim _{n \rightarrow \infty} \frac{3 K\left(\omega_{n}^{-1} \gamma, x\right)+C_{x}}{n} \\
& =\lim _{n \rightarrow \infty} \frac{3 K\left(\gamma, \omega_{n} x\right)+C_{x}}{n}=0 .
\end{aligned}
$$


STEP 2. It follows from Step 1 that there exists $t_{n}>0$ such that

$$
\lim _{n \rightarrow \infty} \frac{K\left(\gamma\left(t_{n}\right), \omega_{n} x\right)}{n}=0
$$

From this and Lemma 2.1,

$$
\begin{aligned}
\lim _{n \rightarrow \infty} \frac{K\left(\omega_{n} x, \gamma\left(t_{n}\right)\right)}{n} & =\lim _{n \rightarrow \infty} \frac{K\left(x, \omega_{n}^{-1} \gamma\left(t_{n}\right)\right)}{n} \\
& \leq 3 \lim _{n \rightarrow \infty} \frac{K\left(\omega_{n}^{-1} \gamma\left(t_{n}\right), x\right)}{n}=0 .
\end{aligned}
$$

Combining (8), (9) and Proposition 4.2, we get

$$
\lim _{n \rightarrow \infty} \frac{K\left(\gamma\left(t_{n}\right), x\right)}{n} \leq \lim _{n \rightarrow \infty} \frac{K\left(\gamma\left(t_{n}\right), \omega_{n} x\right)+K\left(\omega_{n} x, x\right)}{n}=A,
$$

and

$$
\lim _{n \rightarrow \infty} \frac{K\left(\gamma\left(t_{n}\right), x\right)}{n} \geq \lim _{n \rightarrow \infty} \frac{-K\left(\omega_{n} x, \gamma\left(t_{n}\right)\right)+K\left(\omega_{n} x, x\right)}{n}=A
$$

Hence,

$$
\lim _{n \rightarrow \infty} \frac{K\left(\gamma\left(t_{n}\right), x\right)}{n}=A
$$

Further,

$$
\lim _{n \rightarrow \infty} \frac{K\left(\gamma\left(t_{n}\right), \gamma(0)\right)}{n}=A
$$

By the assumption of symmetric finite first moment, for $\mathbf{P}$-a.e. sample path $\left\{\omega_{n} x\right\}_{n=1}^{\infty}$,

$$
\lim _{n \rightarrow \infty} \frac{K\left(\omega_{n} x, \omega_{n+1} x\right)}{n}=0, \quad \lim _{n \rightarrow \infty} \frac{K\left(\omega_{n+1} x, \omega_{n} x\right)}{n}=0
$$

Hence

$$
\lim _{n \rightarrow \infty} \frac{K\left(\gamma\left(t_{n}\right), \gamma\left(t_{n+1}\right)\right)}{n}=0, \quad \lim _{n \rightarrow \infty} \frac{\left.K\left(\gamma\left(t_{n+1}\right)\right), \gamma\left(t_{n}\right)\right)}{n}=0 .
$$

To finish the proof, it suffices to show that there exists $N>0$ such that either $t_{n}>0$ for all $n>N$ or $t_{n}<0$ for all $n>N$. Suppose this is not true. Then there are infinitely many $n_{k}$ such that $t_{n_{k}}<0$ and $t_{n_{k}+1}>0$. As a consequence,

$$
K\left(\gamma\left(t_{n_{k}}\right), \gamma\left(t_{n_{k}+1}\right)\right)=K\left(\gamma\left(t_{n_{k}}\right), \gamma(0)\right)+K\left(\gamma(0), \gamma\left(t_{n_{k}+1}\right)\right)>K\left(\gamma\left(t_{n_{k}}\right), \gamma(0)\right) .
$$


Further

$$
\lim _{k \rightarrow \infty} \frac{K\left(\gamma\left(t_{n_{k}}\right), \gamma\left(t_{n_{k}+1}\right)\right)}{n_{k}} \geq \lim _{k \rightarrow \infty} \frac{K\left(\gamma\left(t_{n_{k}}\right), \gamma(0)\right)}{n_{k}}=A
$$

which contradicts $(10)$.

\section{REFERENCES}

[1] F. Bonahon, Geodesic laminations on surfaces, Laminations and foliations in dynamics, geometry and topology, Contemp. Math. 269, Amer. Math. Soc., Providence, RI, 2001, 1-37.

[2] P. BUSER, Geometry and spectra of compact Riemann surfaces, Reprint of the 1992 ed., Modern Birkhäuser Classics, Birkhäuser Boston, Boston, MA, 2010.

[3] A. Casson and S. A. Bleiler, Automorphisms of surfaces after Nielsen and Thurston, Cambridge University Press, 1988.

[4] Y. Chol AND K. RaFI, Comparison between Teichmüller and Lipschitz metrics, J. Lond. Math. Soc. 76 (2007), 739-756.

[5] A. Fathi, F. Laudenbach and V. PoÉnaru, Thurston's work on surfaces, translated from French by Djun Kim and Dan Margalit, Mathematical Notes, Princeton University Press, 2013.

[6] C. Horbez, Central limit theorems for mapping class groups and $\operatorname{Out}\left(F_{n}\right)$. arXiv preprint arXiv:1506.07244, 2015.

[ 7 ] V. A. Kaimanovich AND H. Masur, The poisson boundary of the mapping class group, Invent. Math. 125 (1996), 221-264.

[8] V. A. Kaimanovich, The Poisson formula for groups with hyperbolic properties, Ann. of Math. 152 (2000), 659-692.

[9] A. Karlsson and F. Ledrappier, Linear drift and possion boundary for random walks, Pure and Applied Mathematics Quarterly 3 (2007), 1027-1036.

[10] A. KARLSSON, Two extensions of Thurston's spectral theorem for surface diffeomorphisms, Bull. Lond. Math. Soc. 46 (2014), 217-226.

[11] G. LevitT, Foliations and laminations on hyperbolic surfaces, Topology 22 (1983), 119135.

[12] Z. LI, Length spectrums of Riemann surfaces and the Teichmüller metric, Bull. London Math. Soc. 35 (2003), 802-810.

[13] L. LiU, Z. Sun And H. WeI, Topological equivalence of metrics in Teichmüller space, Anna. Acad. Sci. Fenn. Math. 33 (2008), 159-170.

[14] A. Papadopoulos, On Thurston's boundary of Teichmüller space and the extension of earthquakes, Topology and its Applications 41 (1991), 147-177.

[15] A. Papadopoulos and G. Thret, On the topology defined by Thurston's asymmetric metric, Math. Proc. Cambridge Philos. Soc. 142 (2007), 487-496.

[16] R. C. PENNER AND J. L. HaRer, Combinatorics of train tracks, Annals of mathematics studies 125, Princeton University Press, 1992.

[17] G. ThÉTET, A propos de la métrique asymétrique de Thurston sur l'espace de Teichmüller d'une surface, preprint.

[18] G. Tiozzo, Sublinear deviation between geodesics and sample paths, Duke Math. J. 164 (2015), 511-539.

[19] W. P. Thurston, Minimal stretch maps between hyperbolic surfaces, arXiv:math/9801039v1. 
[20] C. WALSH, The horoboundary and isometry group of Thurstons Lipschitz metric, Handbook of Teichmüller Theory IV, 2014, 327-353.

Huiping Pan School of Mathematics and Computational Science Sun Yat-SEN University

510275, GuANGZHou

P. R. CHINA

E-mail: chnpanhp@foxmail.com 